\title{
Revascularização do miocárdio sem circulação extracorpórea: experiência e resultados iniciais
}

\author{
Luiz Antônio BRASIL*, João Batista MARIANO*, Fernando Martins dos SANTOS*, \\ André Luiz SILVEIRA*, Nilo de MELO*, Nivaldo Gomes de OLIVEIRA*, \\ Rômulo Sales ANDRADE*, Delzirene Pinheiro BOTELHO*, Antônio CALZADA*
}

RBCCV 44205-481

\begin{abstract}
Brasil L A, Mariano J B, Santos F M, Silveira A L, Melo N, Oliveira N G, Andrade R S, Botelho D P, Calzada A - Revascularização do miocárdio sem circulação extracorpórea: experiência e resultados iniciais. Rev Bras Cir Cardiovasc 2000; 15(1): 6-15.
\end{abstract}

RESUMO: Fundamentos: A operação de revascularização do miocárdio sem circulação extracorpórea (CEC) vem sendo utilizada como uma alternativa para o tratamento da insuficiência coronariana.

Objetivo: Apresentar nossa experiência com este procedimento, descrevendo a técnica empregada e os resultados iniciais.

Casuística e Métodos: Foram avaliados 23 pacientes submetidos à revascularização do miocárdio sem CEC. Foram selecionados para este estudo pacientes que apresentavam lesões nas artérias coronárias da região ântero-diafragmática do coração. A principal indicação cirúrgica foi insuficiência coronária crônica $(78,3 \%)$. O sexo masculino predominou em $65 \%$ dos casos. A idade variou de 44 a 80 anos (média: 59,6 anos). A abordagem cirúrgica em todos os pacientes foi através de esternotomia mediana. Os enxertos utilizados foram: as artérias torácicas internas, veia safena e artéria radial.

Resultados: O tempo médio de operação foi de $3 \mathrm{~h} 15 \mathrm{~min}$. Não houve intercorrências intra-operatórias. O número de enxertos por paciente variou de 1 a 3, num total de 36 enxertos realizados, com média de 1,56 enxerto/paciente. A artéria torácica interna esquerda foi o enxerto mais utilizado $(41,7 \%)$. As artérias coronárias revascularizadas mais freqüentemente foram o ramo interventricular anterior $(52,8 \%)$ e a coronária direita $(30,5 \%)$. A mortalidade hospitalar e a incidência de infarto pós-operatório foram de 4,3\%. Não ocorreram complicações neurológicas, pulmonares, renais, hemorrágicas ou infecciosas. O tempo médio de internação hospitalar foi de 7 dias.

Conclusão: A revascularização do miocárdio sem CEC é uma técnica eficaz e segura que pode ser realizada em casos selecionados, com baixa morbidade e mortalidade, com redução de custos e do tempo de internação hospitalar.

DESCRITORES: Revascularização miocárdica. Circulação extracorpórea. Cardiopatias, cirurgia.

\section{INTRODUÇÃO}

A revascularização cirúrgica do miocárdio se consagrou nestes últimos 30 anos como um procedimento seguro e bem estabelecido para o tratamento da insuficiência coronariana, proporcionando a remissão dos sintomas e contribuindo para o prolongamento e melhora da qualidade de vida de pacientes portadores de doença coronária.

\footnotetext{
Trabalho realizado na Santa Casa de Misericórdia de Goiânia. Goiânia, GO, Brasil.

* Da Santa Casa de Misericórdia de Goiânia.

Recebido para publicação março de 1999.

Endereço para correspondência: Luiz Antonio Brasil. Rua T-53, № 235 - Quadra H-22 - Lote 05, Setor Marista. Goiânia, GO, Brasil. CEP $74150-310$. Tel: (062) 241-3997. e-mail: labrasil@cardiol.br
} 
Brasil L A, Mariano J B, Santos F M, Silveira A L, Melo N, Oliveira N G, Andrade R S, Botelho D P, Calzada A - Revascularização do miocárdio sem circulação extracorpórea: experiência e resultados iniciais. Rev Bras Cir Cardiovasc 2000; 15 (1): 6-15.

Apesar dos baixos índices de mortalidade hospitalar, a morbidade pós-operatória ainda permanece considerável, especialmente para aqueles pacientes com doenças associadas tais como: renais crônicos, portadores de doença pulmonar obstrutiva crônica (DPOC), presença de acidente vascular cerebral prévio, distúrbios de coagulação, doenças metabólicas e endócrinas ou, ainda, pacientes com faixa etária elevada.

Esta morbidade é causada principalmente pelos efeitos sistêmicos adversos decorrentes do uso da circulação extracorpórea (CEC) $(1,2)$.

Atualmente, existe uma tendência a minimizar a agressão ao paciente, simplificando 0 ato cirúrgico através de técnicas menos invasivas, com a finalidade de acelerar a recuperação dos pacientes, diminuir o tempo de hospitalização, os custos e a morbimortalidade. Importante passo nesse sentido foi dado pela introdução das técnicas de revascularização do miocárdio sem o uso da circulação extracorpórea.

A idéia de revascularizar o miocárdio com o coração batendo surgiu com CARREL ${ }^{(3)}$, em 1910. trabalhando experimentalmente. Nos anos 50, esta idéia foi revivida por MURRAY et al. (4), apesar de VINEBERG ${ }^{(5)}$, em 1946, ter introduzido o implante da artéria mamária interna diretamente no miocárdio.

A revascularização do miocárdio sem circulação extracorpórea foi realizada com sucesso, inicialmente, em 1967 por KOLESSOV (6), na União Soviética, e por FAVALORO (7) em 1968 e GARRETT et al. (8) em 1973, nos Estados Unidos.

Em 1975, TRAPP \& BISARYA (9), no Canadá, e ANKENEY (10), nos Estados Unidos, registraram seus resultados com grandes grupos de pacientes revascularizados sem assistência da CEC. Estes trabalhos foram interrompidos pela difusão da técnica preconizada por FAVALORO (11), que revolucionou a operação de revascularização do miocárdio, proporcionando excelentes condições operatórias para a realização das anastomoses safeno-coronárias com o coração parado, sob circulação extracorpórea.

Entusiasmado com o progresso farmacológico pelo surgimento de drogas que diminuíam o consumo de oxigênio do miocárdio, BUFFOLO et al. (12-15) iniciaram no Brasil, em 1981, a operação de revascularização do miocárdio, sem circulação extracorpórea, sendo responsáveis pela introdução e padronização da técnica em nosso meio, sugerindo seu uso sistemático como uma alternativa eficiente e segura no tratamento da insuficiência coronariana.

Outros autores como BENETTI (16), na Argentina, e ARCHER et al. (17), nos Estados Unidos, demonstraram as vantagens da técnica e incorporaram esta alternativa aos procedimentos de revascularização do miocárdio.
Em nosso país, outros Serviços têm registrado suas experiências, demonstrando que a revascularização do miocárdio sem CEC é um procedimento que pode ser realizado com baixo risco cirúrgico e com excelentes resultados (18-20). Como fatores determinantes podemos citar a melhora do instrumental cirúrgico, das técnicas anestésicas, dos cuidados pós-operatórios e, principalmente, do aperfeiçoamento (treinamento) das equipes cirúrgicas com este procedimento.

O objetivo deste trabalho é apresentar a nossa experiência com a revascularização do miocárdio sem circulação extracorpórea, descrevendo a técnica empregada e nossos resultados iniciais.

\section{CASUÍSTICA E MÉTODOS}

Foram operados 23 pacientes, portadores de insuficiência coronariana, com a técnica de revascularização do miocárdio sem circulação extracorpórea.

Os pacientes foram selecionados obedecendo os seguintes critérios:

Pacientes que apresentavam lesões em artérias de fácil acesso, localizadas na região ânterodiafragmática do coração: ramo interventricular anterior (RIA), artéria diagonal e coronária direita.

Foram excluídos os pacientes que apresentavam lesões no território da circunflexa nesta fase inicial de nossa experiência, pois, na maioria dos casos, há necessidade de luxação do coração o que pode induzir à instabilidade hemodinâmica, com presença de hipotensão arterial, distensão do coração, ocasionadas pela torsão dos grandes vasos da base.

Também foram excluídos os pacientes com artérias coronárias de trajeto intramiocárdico, pacientes com artérias de fino calibre, portadores de coronárias com intensa ateromatose parietal, ou ainda pacientes com dependência total da artéria a ser tratada, uma vez que sua interrupção, mesmo temporária, seria de alto risco.

Nenhum dos pacientes desta série havia sido submetido previamente à operação cardíaca.

A indicação cirúrgica se baseou nas seguintes condições clínicas: 18 (78,3\%) pacientes eram portadores de insuficiência coronária crônica, 2 (8,7\%) apresentavam angina instável, $1(4,3 \%)$ estava com quadro de infarto agudo em evolução e 2 (8,7\%) foram para a operação após insucesso da angioplastia (Tabela 1).

Com relação a doenças associadas 5 (21,7\%) pacientes apresentavam enfisema pulmonar de grau avançado, $2(8,7 \%)$ eram portadores de doenças renais crônicas e faziam uso de hemodiálise, 4 
Brasil L A, Mariano J B, Santos F M, Silveira A L, Melo N, Oliveira N G, Andrade R S, Botelho D P, Calzada A - Revascularização do miocárdio sem circulação extracorpórea: experiência e resultados iniciais. Rev Bras Cir Cardiovasc 2000; 15 (1): 6-15.

TABELA 1

INDICAÇÕES CIRÚRGICAS

\begin{tabular}{lrr}
\hline & N & $\%$ \\
\hline Insuficiência coronária crônica & 18 & 78,3 \\
Angina instável & 2 & 8,7 \\
Infarto agudo em evolução & 1 & 4,3 \\
Pós-angioplastia sem sucesso & 2 & 8,7 \\
\hline & $\mathbf{2 3}$ & $\mathbf{1 0 0}$ \\
\hline
\end{tabular}

$(17,3 \%)$ tinham sofrido infarto agudo do miocárdio prévio, 4 (17,3\%) eram diabéticos tipo II e 13 (56,5\%) eram portadores de hipertensão arterial sistêmica (Tabela 2).

Dos 23 pacientes, 15 (65\%) eram do sexo masculino e 8 (35\%) do sexo feminino (Tabela 3$)$.

A idade variou de 44 a 80 anos, com média de 59,6 anos, sendo que $4(17,3 \%)$ pacientes com idade igual ou superior a 70 anos (Tabela 3 ).

Em $12(52,2 \%)$ pacientes a lesão coronária era única, em $9(39,1 \%)$ havia comprometimento de dois
TABELA 2

DOENÇAS ASSOCIADAS

\begin{tabular}{lrr}
\hline & N & $\%$ \\
\hline Enfisema pulmonar & 5 & 21,7 \\
Insuficiência renal crônica & 2 & 8,7 \\
Infarto do miocárdio prévio & 4 & 17,3 \\
Diabete melito & 4 & 17,3 \\
Hipertensão arterial sistêmica & 13 & 56,5 \\
\hline
\end{tabular}

vasos e $2(8,7 \%)$ apresentavam três artérias comprometidas (Tabela 3 ).

Os enxertos utilizados para revascularizar o coração foram os seguintes: artéria torácica interna esquerda (ATIE), artéria torácica interna direita (ATID), artéria radial e veia safena (Tabela 3 ).

\section{Técnica Operatória}

Após a monitorização eletrocardiográfica da pressão arterial média, da pressão venosa central, do

TABELA 3

CARACTERIZAÇÃO DOS PACIENTES QUANTO AO SEXO, IDADE, ARTÉRIAS COMPROMETIDAS E ENXERTO UTILIZADO

\begin{tabular}{|c|c|c|c|c|}
\hline PACIENTE & SEXO & IDADE & $\begin{array}{c}\text { ARTÉRIAS } \\
\text { COMPROMETIDAS }\end{array}$ & $\begin{array}{l}\text { ENXERTO } \\
\text { UTILIZADO }\end{array}$ \\
\hline 1 & M & 56 & RIA, CD & ATIE + RAD \\
\hline 2 & M & 60 & CD & ATID \\
\hline 3 & $\mathrm{~F}$ & 61 & RIA & ATIE \\
\hline 4 & M & 63 & $\mathrm{RIA}, \mathrm{CD}$ & $S A F+S A F$ \\
\hline 5 & $M$ & 44 & RIA & ATIE \\
\hline 6 & $\mathrm{~F}$ & 52 & RIA, CD & ATIE + ATID \\
\hline 7 & $M$ & 54 & RIA & ATIE \\
\hline 8 & M & 80 & RIA & SAF \\
\hline 9 & $M$ & 70 & RIA, Dg & ATIE + SAF \\
\hline 10 & $\mathrm{~F}$ & 64 & RIA, CD & $S A F+S A F$ \\
\hline 11 & $\mathrm{~F}$ & 66 & CD & SAF \\
\hline 12 & $M$ & 75 & RIA & SAF \\
\hline 13 & $\mathrm{~F}$ & 55 & $\mathrm{RIA}, \mathrm{Dg}$ & ATIE + RAD \\
\hline 14 & $\mathrm{~F}$ & 48 & RIA & ATIE \\
\hline 15 & $M$ & 50 & RIA, Dg & ATIE + RAD \\
\hline 16 & M & 56 & RIA & ATIE \\
\hline 17 & M & 62 & $\mathrm{RIA}, \mathrm{Dg}, \mathrm{CD}$ & ATIE + SAF + SAF \\
\hline 18 & M & 68 & CD & SAF \\
\hline 19 & M & 59 & RIA, Dg, CD & $A T I E+S A F+S A F$ \\
\hline 20 & M & 71 & CD & SAF \\
\hline 21 & $\mathrm{~F}$ & 53 & RIA & ATIE \\
\hline 22 & M & 50 & RIA, CD & ATIE + RAD \\
\hline 23 & $\mathrm{~F}$ & 55 & $\mathrm{RIA}, \mathrm{Dg}$ & ATIE + RAD \\
\hline \multicolumn{5}{|c|}{$M=15 ; F=8 ;$ Média = 59,6 } \\
\hline
\end{tabular}


Brasil L A, Mariano J B, Santos F M, Silveira A L, Melo N, Oliveira N G, Andrade R S, Botelho D P, Calzada A - Revascularização do miocárdio sem circulação extracorpórea: experiência e resultados iniciais.

débito urinário e oximetria de pulso, o paciente foi colocado em decúbito dorsal e foi anestesiado. A anestesia não diferiu da utilizada nos pacientes operados com CEC. Em seguida à indução com neuroleptoanalgesia, o paciente foi intubado e mantido sob ventilação mecânica com oxigênio a 100\%, tomando-se o cuidado de manter as funções hemodinâmica e respiratória estáveis durante todo o ato operatório.

A abordagem cirúrgica em todos os pacientes foi realizada através de toracotomia mediana reduzida, isto é, incisionando a pele, subcutâneo e tecido muscular, superiormente, ao nível da junção do manúbrio com o corpo do esterno e, inferiormente, na junção do corpo esternal com o processo xifóide. Em seguida, realizamos a secção do esterno (esternotomia) longitudinal total, proporcionando boa exposição do coração e permitindo a dissecção da artéria torácica interna esquerda ou direita em toda sua extensão. O coração foi então exposto através

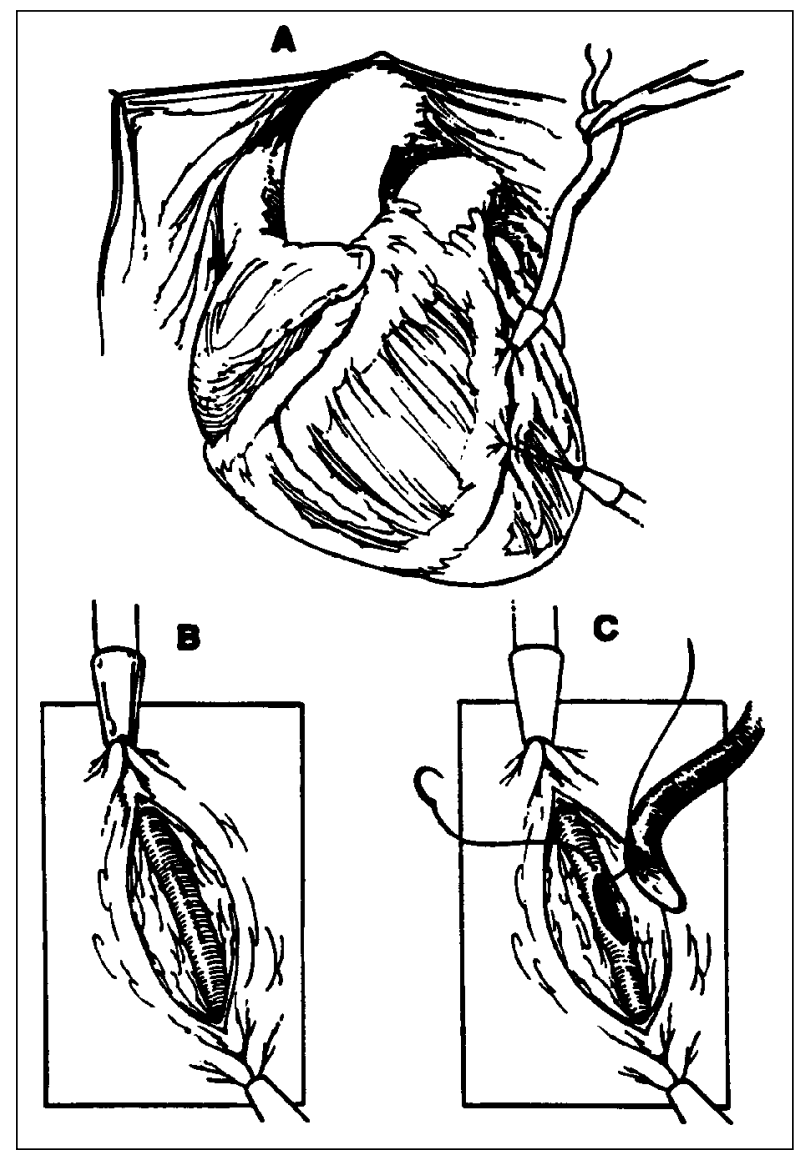

Fig. 1 - A: Identificada a artéria a ser revascularizada, fios de prolene 5-0 são passados, proximal e distalmente, em torno da coronária. B: A coronária é dissecada e garroteada, proximal e distalmente, através de torniquetes delicados de silicone. C: Abertura longitudinal da coronária. Realização da anastomose com sutura contínua usando fio de prolene 7-0. da fixação do pericárdio aos campos cirúrgicos e luxado com compressas para melhor exposição e estabilização das artérias a serem revascularizadas. Uma vez identificada a artéria a ser revascularizada, escolhemos e dissecamos a área de anastomose; fios de prolene 5-0 foram passados proximal e distalmente em torno da coronária. Ressaltamos que tanto o perfusionista como todo o equipamento para a imediata instalação da circulação extracorpórea se encontravam na sala, à disposição da equipe cirúrgica.

Estando todos os enxertos a serem utilizados preparados, procedeu-se à heparinização sistêmica do paciente na dose de $2 \mathrm{mg} / \mathrm{kg}$ mantendo-se esta heparinização com a metade da dose inicial, a cada hora. A freqüência cardíaca foi previamente reduzida com uso de Verapamil (5mg EV). A artéria coronária foi então garroteada, proximal e distalmente, através de torniquetes delicados de silicone. Em seguida, procedemos à abertura longitudinal da coronária e realizamos a anastomose com sutura contínua usando fio de prolene 7-0 (Figuras 1 e 2).

Durante a confecção da anastomose utilizamos,

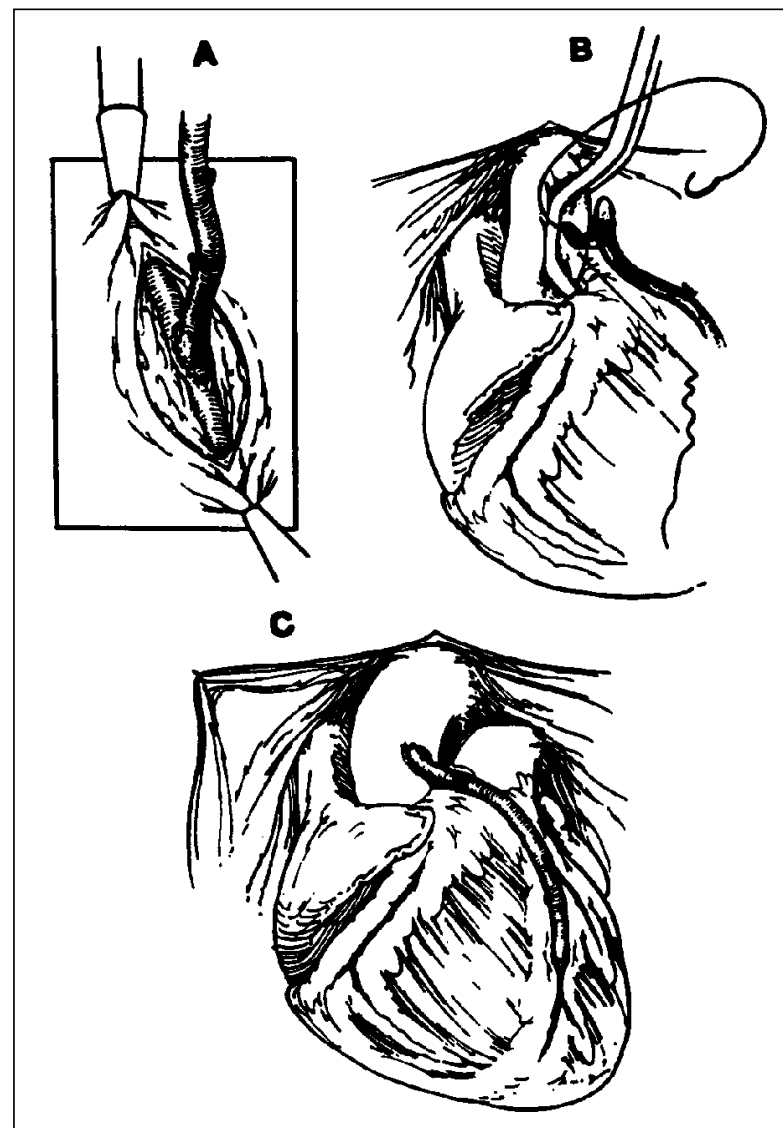

Fig. 2 - D: Anastomose distal realizada. E: Pinçamento tangencial da aorta ascendente para realização da anastomose proximal com fio de prolene 6-0. F: Enxerto aorto-coronário concluído. 
Brasil L A, Mariano J B, Santos F M, Silveira A L, Melo N, Oliveira N G, Andrade R S, Botelho D P, Calzada A - Revascularização do miocárdio sem circulação extracorpórea: experiência e resultados iniciais. Rev Bras Cir Cardiovasc 2000; 15 (1): 6-15.

se necessária, irrigação local através de uma seringa com soro morno para facilitar sua realização.

Antes de terminar a anastomose, testamos a patência da mesma liberando os garrotes distal e proximal e observamos o fluxo sangüíneo local.

Utilizamos também dilatadores de coronária (bugias), proximal e distalmente à anastomose, com o cuidado de ultrapassar os locais de garroteamento, evitando-se com isto estenoses residuais nas coronárias, produzidas pelo garroteamento.

Quando utilizamos como enxerto veia safena ou artéria radial, a anastomose proximal foi realizada, preferencialmente, na aorta ascendente através de pinçamento tangencial da mesma e sutura contínua com fio de prolene 6-0, evitando-se locais com presença de placas de ateroma (Figura 2).

Completadas todas as anastomoses, procedeuse à neutralização da heparina, com infusão lenta EV de cloridrato de protamina, na dose de 1:1, diluída em soro glicosado a $5 \%$.

Após a revisão de hemostasia, deixamos 2 fios de marcapasso epicárdicos temporários, realizamos a drenagem do mediastino e, finalmente, o fechamento da toracotomia (esternotomia) por planos.

\section{RESULTADOS}

Em todos os casos o ato operatório transcorreu sem qualquer tipo de intercorrência intra-operatória, com ausência de alterações hemodinâmicas ou arritmias durante a realização das anastomoses.

O tempo total de operação variou de 2 h30 min a $4 \mathrm{~h}$ com média de $3 \mathrm{~h} 15 \mathrm{~min}$.

O número de enxertos realizados por paciente variou de 1 a 3 , tendo $12(52,2 \%)$ recebido um enxerto, 9 (39,1\%) dois enxertos e $2(8,7 \%)$ três enxertos, num total de 36 enxertos realizados, com média de 1,56 enxerto/paciente.

O tempo total médio de isquemia para a realização das anastomoses foi de $16 \mathrm{~min}$, não sendo utilizado nenhum tipo de estabilizador de sutura, somente compressas para fixar o coração e expor a artéria a ser revascularizada.

As coronárias revascularizadas mais freqüentemente foram o ramo interventricular anterior $(52,8 \%)$ e a coronária direita $(30,5 \%)$ - Tabela 4 .

Quanto ao tipo de enxerto utilizado, a artéria torácica interna esquerda (ATIE) foi empregada 15 $(41,7 \%)$ vezes e a artéria torácica interna direita (ATID) 2 (5,5\%) vezes. Em todas as ocasiões, tanto a ATIE como a ATID foram usadas como enxertos pediculados. Em 14 (38,9\%) pontes aorto-coronárias foi utilizada a veia safena. A artéria radial também foi empregada como enxerto aorto-coronário 5 $(13,9 \%)$ vezes (Tabela 5 ).

Com relação à artéria revascularizada e ao enxerto utilizado, nas 19 revascularizações do ramo interventricular anterior, a ATIE foi o enxerto mais usado - $15(78,9 \%)$ vezes. Em 4 (21,1\%) situações empregamos a veia safena para revascularizar o ramo interventricular anterior. A coronária direita foi revascularizada mais freqüentemente com veia safena - $7(63,6 \%)$ casos. Em $2(18,2 \%)$ casos foi utilizada a ATID e em outros 2 casos, a artéria radial. Já a artéria diagonal foi revascularizada igualmente através de veia safena e artéria radial (Tabela 6).

A mortalidade hospitalar foi de $4,3 \%$, ocorrendo óbito em nossa casuística, de um paciente considerado de alto risco, que foi operado com infarto anterior extenso em evolução. Este paciente era diabético, hipertenso e apresentava dor precordial em repouso. Foi estudado no $4^{\circ}$ dia pós-IAM e lesão crítica segmentar na origem do ramo interventricular anterior, associada com disfunção ventricular importante, foi diagnosticada. Após a revascularização, o paciente apresentou boa evolução pós-operatória, com desaparecimento dos sintomas e estabilidade hemodinâmica até o $9^{\circ} \mathrm{PO}$, quando iniciou com arritmia ventricular, que se tornou incontrolável clinicamente, culminando com óbito no $12^{\circ} \mathrm{PO}$.

Como complicação não fatal, 1 (4,3\%) paciente apresentou infarto agudo do miocárdio (IAM) no pósoperatório (1ำ PO), com presença de alteração eletrocardiográfica e enzimática, sem repercussão hemodinâmica. Este paciente possuía lesões nas

TABELA 4

ARTÉRIAS CORONÁRIAS REVASCULARIZADAS

\begin{tabular}{lrr}
\hline & $\boldsymbol{N}$ & $\%$ \\
\hline Ramo interventricular anterior & 19 & 52,8 \\
Coronária direita & 11 & 30,5 \\
Diagonal & 6 & 16,7 \\
\hline Total & $\mathbf{3 6}$ & $\mathbf{1 0 0 , 0}$ \\
\hline
\end{tabular}

TABELA 5

TIPO DE ENXERTO UTILIZADO

\begin{tabular}{lrr}
\hline & N & $\%$ \\
\hline Artéria torácica interna esquerda & 15 & 41,7 \\
Veia safena & 14 & 38,9 \\
Artéria radial & 5 & 13,9 \\
Artéria torácica interna direita & 2 & 5,5 \\
\hline Total & $\mathbf{3 6}$ & $\mathbf{1 0 0 , 0}$ \\
\hline
\end{tabular}


Brasil L A, Mariano J B, Santos F M, Silveira A L, Melo N, Oliveira N G, Andrade R S, Botelho D P, Calzada A - Revascularização do miocárdio sem circulação extracorpórea: experiência e resultados iniciais. Rev Bras Cir Cardiovasc 2000; 15 (1): 6-15.

TABELA 6

ARTÉRIA REVASCULARIZADA X TIPO DE ENXERTO UTILIZADO

\begin{tabular}{|c|c|c|c|c|c|c|c|c|c|c|}
\hline & \multicolumn{2}{|c|}{ ATIE } & \multicolumn{2}{|c|}{ SAFENA } & \multicolumn{2}{|c|}{ RADIAL } & \multicolumn{2}{|c|}{ ATID } & \multicolumn{2}{|c|}{ TOTAL } \\
\hline & $N$ & $\%$ & $N$ & $\%$ & $N$ & $\%$ & $N$ & $\%$ & $N$ & $\%$ \\
\hline $\begin{array}{l}\text { Ramo Interventricular } \\
\text { Anterior }\end{array}$ & 15 & 78,9 & 4 & 21,1 & 一 & - & - & - & 19 & 100 \\
\hline Coronária Direita & - & - & 7 & 63,6 & 2 & 18,2 & 2 & 18,2 & 11 & 100 \\
\hline Diagonal & - & - & 3 & 50,0 & 3 & 50,0 & - & - & 6 & 100 \\
\hline Total Enxertos & \multicolumn{2}{|c|}{15} & \multicolumn{2}{|c|}{14} & \multicolumn{2}{|c|}{5} & \multicolumn{2}{|c|}{2} & 36 & 100 \\
\hline
\end{tabular}

ATIE = Artéria Torácica Interna Esquerda; ATID = Artéria Torácica Interna Direita

origens do ramo interventricular anterior, que era fino e tortuoso e da diagonal que era bem calibrosa. Foi revascularizado com ATIE para o ramo interventricular anterior e veia safena para a diagonal. Este resultado insatisfatório foi provavelmente pelo uso da ATIE que também era de fino calibre, o que causou dificuldade técnica para a realização da anastomose.

Todos os outros pacientes apresentaram boa evolução pós-operatória, com ausência de alterações eletrocardiográficas ou enzimáticas que pudessem caracterizar infarto intra e pós-operatório.

O índice de complicações cardíacas com presença de arritmias e instabilidade hemodinâmica foi mínimo, não havendo com isso necessidade do uso de drogas no pós-operatório, principalmente inotrópicas positivas.

Não tivemos, em nenhum dos casos, complicações neurológicas, pulmonares, renais, hemorrágicas ou infecciosas.

Destes 23 pacientes operados sem CEC, 8 $(34,7 \%)$ foram extubados na sala cirúrgica, após o término da operação, fato que contribuiu para abreviar o tempo de permanência dos mesmos na UTI. Os outros 15 pacientes permaneceram na UTI por 48 horas, sendo extubados, em média, com 8 horas de pós-operatório.

O tempo de internação hospitalar variou de 6 a 12 dias (média de 7 dias).

\section{COMENTÁRIOS}

A circulação extracorpórea é indispensável na maioria das operações cardíacas, sendo responsável pelo enorme progresso de nossa especialidade. Porém, ainda é um procedimento que não está isento de complicações. Pacientes que são submetidos à operação cardíaca com uso de CEC podem desenvolver uma síndrome de resposta inflamatória sistêmica (SRIS) de graus variáveis, produzindo lesões orgânicas que levam ao aumento da morbidade pósoperatória. Como fatores causais desta reação inflamatória, também denominada de síndrome pós-perfusão, podemos citar o trauma cirúrgico, o contato do sangue com superfícies não endotelizadas do circuito extracorpóreo e as chamadas lesões de reperfusão pós-CEC (21). Ocorre ativação de elementos do sangue provocando uma resposta humoral e celular, com liberação de mediadores responsáveis pelo aparecimento de febre não infecciosa, leucocitose, aumento da permeabilidade capilar, acúmulo de líquido intersticial, alterações da coagulação e disfunção de órgãos, principalmente em território cardíaco e pulmonar (22).

Os riscos das operações de coronária têm aumentado nos últimos anos devido ao avanço das técnicas de angioplastia, que seleciona os melhores pacientes, pelo número crescente de reoperações e também pela ampliação das indicações a determinados grupos de pacientes, como idosos e portadores de doenças sistêmicas associadas (23).

Podemos dizer, com certeza, que a principal causa responsável pela diminuição da morbidade e mortalidade das cirurgias coronarianas, principalmente nestes grupos de pacientes, foi sem dúvida a introdução da técnica de revascularização do miocárdio sem utilização da circulação extracorpórea.

Recentemente, BUFFOLO et al. (24) demonstraram que este tipo de operação pode ser realizado com baixa morbidade e mortalidade, principalmente nos grupos considerados de risco e divulgaram os principais critérios de indicação deste procedimento, relacionando suas vantagens e desvantagens com a revascularização convencional.

Em estudo recentemente publicado (25), tivemos a oportunidade de comparar pacientes portadores de insuficiência coronariana submetidos à revascularização do miocárdio com e sem CEC. O grupo de doentes que foi revascularizado sem CEC apresentou menor liberação de citocinas (TNFa) e evoluiu mais estável hemodinamicamente, com menos hipotensão arterial, menor uso de drogas 
Brasil L A, Mariano J B, Santos F M, Silveira A L, Melo N, Oliveira N G, Andrade R S, Botelho D P, Calzada A - Revascularização do miocárdio sem circulação extracorpórea: experiência e resultados iniciais. Rev Bras Cir Cardiovasc 2000; 15 (1): 6-15.

vasoativas, freqüência cardíaca menos elevada, menor hipertermia, menos leucocitose, menor sangramento pós-operatório e menor tempo de intubação orotraqueal.

Dentre as vantagens da técnica de revascularizar o miocárdio sem CEC constatadas com nossos resultados podemos citar a simplicidade aliada a menor agressividade do ato operatório assemelhando-se ao risco de uma simples toracotomia, o menor tempo cirúrgico, menor uso de sangue e derivados, menor morbi-mortalidade, recuperação mais rápida dos pacientes, menor custo pela não utilização do circuito extracorpóreo e tempo mais curto de internação hospitalar.

Não podemos deixar de ressaltar os inconvenientes e desvantagens que seguramente existem. Um dos pontos de discussão seria sua aplicabilidade em todos os casos, e também nos pacientes com doença multiarterial no sentido de realizar a chamada revascularização completa do coração.

A posição anatômica da artéria coronária a ser revascularizada na superfície do coração é importante, embora a abordagem cirúrgica possa ser modificada. As artérias localizadas posteriormente (ramos laterais da circunflexa) somente são abordáveis se a estabilidade hemodinâmica for mantida enquanto o coração estiver fora de sua posição. A operação se torna tecnicamente difícil se a artéria coronária a ser revascularizada estiver calcificada, intramiocárdica ou se for de calibre reduzido.

Em nosso meio, LOBO FILHO et al. (20) relataram sua experiência com a operação de revascularização completa do miocárdio sem CEC, demonstrando que, atualmente, $99 \%$ dos pacientes de seu Serviço portadores de insuficiência coronariana são revascularizados sem auxílio da CEC.

Outro ponto polêmico relacionado com esta técnica, levantado principalmente pelos grupos que não a utilizam, é com relação à qualidade da anastomose. Salientamos que a realização das anastomoses com o coração batendo, seja com enxertos arteriais ou com veia safena, exige técnica mais apurada e deve ser realizada por cirurgiões que já dominam por completo a operação de revascularização do miocárdio com a técnica convencional (com o coração parado sob circulação extracorpórea) e, para maior segurança, que tenham realizado uma curva de aprendizado em Serviços com larga experiência nessa técnica.

A baixa incidência de infarto pós-operatório em nossos resultados associada à melhora clínica apresentada pelos pacientes, juntamente com os detalhes técnicos descritos realizados ao final das anastomoses nos dão segurança quanto à perviabilidade das anastomoses. A experiência de JATENE et al. (26) publicada recentemente, usando estabilizador de sutura, evidenciou excelentes resultados, proporcionando maior facilidade, segurança e qualidade na confecção das anastomoses.

O controle da freqüência cardíaca durante a operação principalmente no momento da realização da anastomose também é importante e está diretamente relacionado com a conduta anestésica. A anestesia segue os princípios usuais da realizada nos pacientes operados com CEC, mas alguns detalhes podem influir no resultado cirúrgico da revascularização sem CEC, exigindo atenção especial do anestesista.

A manutenção da anestesia, nestes pacientes, requer uma baixa freqüência cardíaca e ausência de contrações vigorosas, para permitir a realização das anastomoses de maneira mais confortável. Com esta finalidade são empregadas drogas que atuam diminuindo a freqüência cardíaca e também o consumo de oxigênio pelo miocárdio, como o verapamil, o diltiazem e os beta-bloqueadores. Dentre os betabloqueadores, o esmolol está sendo muito utilizado em Serviços que praticam a revascularização do miocárdio sem CEC. É um beta-bloqueador cardioseletivo, de curta ação, que reduz a força contrátil do coração e diminui a sua freqüência por curto período de tempo. O esmolol não possui atividade simpaticomimétida, sendo rapidamente eliminado, com meia vida em torno de 9 minutos (27). Esta droga se torna valiosa por suas características no campo da operação cardiovascular, onde o rápido controle da freqüência cardíaca contribui de maneira significativa para a realização das anastomoses com o coração batendo.

Outro ponto importante na técnica de revascularização sem CEC é a isquemia miocárdica induzida no período de realização das anastomoses. O deslocamento do coração para acessar as artérias coronárias, o uso de drogas cronotrópicas negativas e pré-condições isquêmicas podem levar à deterioração hemodinâmica. A monitorização do paciente deve ser rigorosa e incluir ECG, oximetria de pulso, pressão arterial média e, se possível, ecocardiografia transesofágica. A rápida demonstração da isquemia com este aparelho é uma vantagem, pois sua sensibilidade é maior que um ECG (28).

Observações feitas durante a angioplastia coronária demonstraram que a elevação do segmento ST é menor na segunda e subseqüentes oclusões. Um estudo clínico (29) demonstrou que o precondicionamento isquêmico preservou significantemente ATP após 10 minutos de isquemia. As biópsias mostraram que os níveis de ATP eram mais altos no grupo de pacientes que tiveram precondicionamento isquêmico comparados com o grupo controle e, concluiu, que isto pode conferir maior resistência miocárdica ao dano isquêmico. 
Brasil L A, Mariano J B, Santos F M, Silveira A L, Melo N, Oliveira N G, Andrade R S, Botelho D P, Calzada A - Revascularização do miocárdio sem circulação extracorpórea: experiência e resultados iniciais. Rev Bras Cir Cardiovasc 2000; 15 (1): 6-15.

Durante a realização da revascularização do miocárdio sem CEC, o cirurgião precondiciona o miocárdio à isquemia por uma breve, mas controlada oclusão da artéria coronária a ser revascularizada. As oclusões são subletais às células miocárdicas, mas induzem uma rápida adaptação dos miócitos àqueles breves períodos de isquemia e a um atraso na freqüência de morte celular durante o período isquêmico prolongado subseqüente (30). A avaliação do grau de lesão miocárdica durante os testes de oclusão pode ser feita observando-se o grau da pressão na artéria pulmonar, elevação do segmento S-T e movimentos paradoxais da parede segmentar.

Recentemente, BRAILE et al. (31) preconizaram a dosagem de troponina I como marcador de sofrimento miocárdico, demonstrando, através dos níveis séricos de troponina I, menor sofrimento miocárdico pós-revascularização do miocárdio com cirurgia minimamente invasiva, em comparação com o método convencional de revascularização com CEC.

A possibilidade de realizar as anastomoses com o coração batendo e isquemia regional miocárdica com drogas que diminuem o consumo de oxigênio foi demonstrada ser segura para períodos de isquemia de até 21 minutos $(12,24)$.

RIVETTI et al. (32) registraram sua vasta experiência com revascularização do miocárdio sem CEC, usando shunt intracoronário para evitar a isquemia do miocárdio, durante a realização da anastomose entre o enxerto e a artéria coronária.

Acreditamos que os resultados com a revascularização do miocárdio sem CEC poderão diferir entre os grupos em função da padronização da técnica operatória, experiência com o método e dos critérios de indicação.

A maioria dos Serviços que realizam a revascularização do miocárdio sem CEC descrevem a abordagem operatória através de esternotomia mediana, que proporciona total exposição do coração e de todas as suas artérias, com a vantagem, dependendo do caso, de mudança sem transtornos, para a revascularização convencional com uso da CEC.

Há uma preocupação crescente na cirurgia cardíaca, como em todas as demais especialidades cirúrgicas, no sentido de tornar os procedimentos menos invasivos, com o intuito de diminuir custos, morbidade e mortalidade. A colocação de um enxerto em uma artéria nativa com o coração batendo, como já demonstramos, fornece muitas vantagens. Os cuidados intensivos e a permanência hospitalar são diminuídos, assim como a morbidade e mortalidade, com maior risco nas urgências e nas reoperações $(15,33)$.

A utilização da artéria torácica interna esquerda como enxerto pediculado na revascularização do ramo interventricular anterior é um procedimento consagrado com excelentes resultados a curto e longo prazos $(34,35)$.

Ainda hoje não existe consenso de que a cirurgia de artéria coronária minimamente invasiva, conhecida pela sigla MIDCAB (minimally invasive direct coronary artery bypass graft), indicada em casos selecionados com o objetivo de se evitar a CEC e a esternotomia, é um procedimento que pode ser usado como guia para a diminuição da invasibilidade cirúrgica.

A esternotomia é evitada por uma pequena incisão na região infra-mamária, ao nível do $4^{\circ}$ espaço intercostal esquerdo, para realizar enxertos principalmente entre a ATIE e o ramo interventricular anterior. Há possibilidade, em alguns casos, de realização de enxertos compostos para a artéria diagonal, não permitindo porém a abordagem de outros vasos (ex: coronária direita ou ramos da circunflexa) para se realizar uma revascularização completa.

CALAFIORE et al. (36) demonstraram excelentes resultados com esta técnica, enfatizando que $77 \%$ dos pacientes operados através da MIDCAB receberam alta hospitalar no $2^{\circ}$ dia de pós-operatório.

Nos pacientes que são operados através de toracotomia mínima anterior esquerda e, que apresentam lesões em outras artérias que não podem ser tratadas por esta abordagem, tem sido preconizada por alguns Serviços (37) a revascularização destas artérias com angioplastia e colocação de "stents" intracoronários.

Acreditamos que a combinação de operação cardíaca e angioplastia com colocação de "stents" pode levar à redução da permanência em UTI e hospitalar, mas esta complexa estratégia pode resultar num aumento de custo, devido ao número maior de profissionais envolvidos e também ao uso de drogas, materiais e equipamentos excessivamente caros, associados ao tempo prolongado do procedimento.

\section{CONCLUSÕES}

Apesar do pequeno número de pacientes do nosso estudo e do seguimento ainda curto, concluímos que a revascularização do miocárdio sem circulação extracorpórea é uma técnica eficaz no tratamento da insuficiência coronariana, podendo ser reproduzida com segurança pela maioria dos cirurgiões, principalmente em pacientes com lesões nas coronárias da região ântero-diafragmática do coração (ramo interventricular anterior, coronária direita e diagonal), com redução dos custos, baixa morbidade e mortalidade, além da diminuição do tempo de internação hospitalar.

Acreditamos que o futuro desenvolvimento 
da cirurgia cardiovascular poderá permitir aos Serviços a realização de revascularização do miocárdio sem circulação extracorpórea, com alta hospitalar precoce para a maioria dos pacientes portadores de doença coronariana, proporcionando um aumento no número de pacientes beneficiados com esta técnica, incluindo aqueles de alto risco, considerados inoperáveis.

RBCCV 44205-481

Brasil L A, Mariano J B, Santos F M, Silveira A L, Melo N, Oliveira N G, Andrade R S, Botelho D P, Calzada A - Myocardial revascularization without cardiopulmonary bypass: experience and initial results. Rev Bras Cir Cardiovasc 2000; 15(1): 6-15.

ABSTRACT: Background: Myocardial revascularization without cardiopulmonary bypass (CPB) has been used as an alternative for treatment of coronary insufficiency. results.

Objective: To present our experience with this procedure describing the technique used and our initial

Material and Methods: Twenty-three patients were submitted to myocardial revascularization without CPB. The patients selected for this study had lesions in the coronary arteries of the anterodiaphragmatic cardiac region. The main surgical indication was chronic coronary insufficiency $(78.3 \%) .65 \%$ of the patients were male, with age between 44-80 (mean -59.6 years). The surgical approach in all patients was through median sternotomy. He grafts used were internal thoracic arteries, saphenous vein and radial artery.

Results: Mean surgical time was 3:15 hours. There were no intra-operative occurences.

The number of grafts was 1 to 3 in each patient (mean $1.56 \mathrm{graft} /$ patient) out of a total of $36 \mathrm{grafts}$. The left internal thoracic artery was the most used graft (41.7\%). The most frequently revascularized coronary arteries were the anterior interventricular branch $(52.8 \%)$ and the right coronary $(30.5 \%)$. Hospital mortality and postoperative infarct were $4.3 \%$. There were no neurological, pulmonary, renal, hemorragic or infectious complications. The mean hospital stay was 7 days.

Conclusion: Myocardial revascularization without cardiopulmonary bypass is an effective and safe technique that can be utilized in selected cases with low morbidity and mortality, reducing costs and hospital stay.

DESCRIPTORS: Myocardial revascularization. Extracorporeal circulation. Heart disease, surgery.

\section{REFERÊNCIAS BIBLIOGRÁFICAS}

1 Brasil L A - Liberação e efeitos do fator de necrose tumoral alfa (TNF alfa) induzidos pela circulação extracorpórea. [Tese. Mestrado] São Paulo, SP: Escola Paulista de Medicina da Universidade Federal de São Paulo, 1996. 103p.

2 Brasil L A, Gomes W J, Salomão R, Buffolo E - Ativação de citocina (fator de necrose tumoral - a) e resposta clínica induzida pela circulação extracorpórea. Rev Bras Cir Cardiovasc 1996; 11: 188-200.

3 Carrel A - On the experimental surgery of the thoracic aorta heart. Ann Surg 1910; 52: 83-95.

Murray G, Porcheron R, Hilario J, Roschlav W - Anastomosis of the systemic artery to the coronary. Can Med Ass J 1954; 71: 594-7.

5 Vineberg A M - Treatment of coronary insufficiency by implantation of the internal mammary artery into the left ventricular myocardium. J Thorac Surg 1952; 23: 42-54.

6 Kolessov V L - Mammary artery-coronary anastomosis as method of treatment for angina pectoris. $J$ Thorac Cardiovasc Surg 1967; 54: 535-44.

7 Favaloro R G - Saphenous vein autograft replacement of severe segmental coronary artery occlusion: operative technique. Ann Thorac Surg 1968; 5: 334-9.

8 Garrett H E, Dennis E W, DeBakey M E - Aortocoronary bypass with saphenous vein graft: seven years follow up. JAMA 1973; 223: 792-4.

9 Trapp W G \& Bisarya R - Placement of coronary artery bypass graft without pump oxigenator. Ann Thorac Surg 1975; 19: 1-9.

10 Ankeny $\mathrm{J} \mathrm{L} \mathrm{-} \mathrm{To} \mathrm{use} \mathrm{or} \mathrm{not} \mathrm{to} \mathrm{use} \mathrm{the} \mathrm{pump} \mathrm{oxigenator}$ in coronary bypass operations. Ann Thorac Surg 1975; 19: 108-9. (Editorial)

11 Favaloro R G - Saphenous vein graft in the surgical treatment of coronary artery disease: operative technique. J Thorac Cardiovasc Surg 1969; 58: 178-85.

12 Buffolo E, Andrade J C, Succi J E et al. - Revascularização direta do miocárdio sem circulação extracor- 
Brasil L A, Mariano J B, Santos F M, Silveira A L, Melo N, Oliveira N G, Andrade R S, Botelho D P, Calzada A - Revascularização do miocárdio sem circulação extracorpórea: experiência e resultados iniciais. Rev Bras Cir Cardiovasc 2000; 15 (1): 6-15.

pórea: descrição da técnica e resultados iniciais. Arq Bras Cardiol 1982; 38: 365-73.

Buffolo E, Andrade J C, Succi J, Leão L E, Galluci C - Direct myocardial revascularization without cardiopulmonary bypass. Thorac Cardiovasc Surg 1985; 33: $26-9$.

Buffolo E, Andrade J C, Branco J N, Aguiar L F, Ribeiro $E$ E, Jatene A D - Myocardial revascularization without extracorporeal circulation: seven-year experience in 593 cases. Eur J Cardiothorac Surg 1990; 4: 504-8.

Buffolo E, Andrade J C, Branco J N et al. - Revascularização do miocárdio sem circulação extracorpórea: análise dos resultados de 15 anos de experiência. Rev Bras Cir Cardiovasc 1996; 11: 227-31.

Benetti F J - Direct coronary surgery with saphenous vein bypass without either cardiopulmonary bypass or cardiac arrest. J Cardiovasc Surg 1985; 26: 217-22.

Archer R, Ott D A, Parravicini R et al. - Coronary artery revascularization without cardiopulmonary bypass. Texas Heart Inst $J$ 1984; 11: 52-7.

18 Rivetti L A \& Gandra S M A - Revascularização cirúrgica do miocárdio sem auxílio da circulação extracorpórea, com derivação interna temporária. Rev Soc Cardiol Estado de São Paulo 1991; 1: 65-71.

19 Lima R C, Escobar M, Wanderley Neto J et al. - Revascularização do miocárdio sem circulação extracorpórea: resultados imediatos. Rev Bras Cir Cardiovasc 1993; 8: 171-6.

20 Lobo Filho J G, Dantas M C B R, Rolim J G V et al. Cirurgia de revascularização completa do miocárdio sem circulação extracorpórea: uma realidade. Rev Bras Cir Cardiovasc 1997; 12: 115-21.

21 Kirklin J K, Westaby S, Blackstone E H, Kirklin J W, Chenoweth D E, Pacífico A D - Complement and the damaging effects of cardiopulmonary bypass. $J$ Thorac Cardiovasc Surg 1983; 86: 845-57.

Butler J, Rocher G M, Westaby S - Inflammatory response to cardiopulmonary bypass. Ann Thorac Surg 1993; 55: 552-9.

23 Hartz A J, Kuhn E M, Pryor D B et al. - Mortality after coronary angioplasty and coronary artery bypass surgery: the national Medicare experience. Am J Cardiol 1992; 70: 179-85.

24 Buffolo E, Andrade J C S, Branco J N, Teles C A, Aguiar L F, Gomes W J - Coronary artery bypass grafting without cardiopulmonary bypass. Ann Thorac Surg 1996; 61: 63-6.

Brasil L A, Gomes W J, Salomão R, Buffolo E - Inflammatory response after myocardial revascularization

with or without cardiopulmonary bypass. Ann Thorac Surg 1998; 66: 56-9.

Jatene F B, Pêgo-Fernandes P M, Assad R S et al. Cirurgia de revascularização do miocárdio minimamente invasiva: resultados com o uso de videotoracoscopia e do estabilizador de sutura. Rev Bras Cir Cardiovasc 1997; 12: 233-8.

27 Abramson D C, Pivalizza E G, Gottschalk L I - Drug management for coronary revascularization without cardiac standstill: the use of high-dose esmolol. $J$ Cardiothorac Vasc Anesth 1995; 9: 184-8.

28 Ellis J E, Shah M N, Briller J E, Roizen M F, Aronson $S$, Feinstein S B - A comparison of methods for the detection of myocardial ischemia during noncardiac surgery: automated ST-segment analysis systems, electrocardiography, and transesophageal echocardiography. Anesth Analg 1992; 75: 764-72.

29 Alkhulaifi A M, Yellon D M, Pugsley W B - Preconditioning the human heart during aorto-coronary bypass surgery. Eur J Cardiothorac Surg 1994; 8: 270-6.

30 Murry C E, Jennings R B, Reimer K A - Preconditioning with ischemia a delay of lethal cell injury in ischaemic myocardium. Circulation 1986; 74: 1124-36.

31 Braile D M, Leal J C F, Soares M J et al. - Revascularização do miocárdio com cirurgia minimamente invasiva (MIDCAB): resultados em 46 pacientes. Rev Bras Cir Cardovasc 1998; 13: 194-7.

32 Rivetti L A, Gandra S M A, Silva A M R P, Campagnucci V P - Revascularização do miocárdio sem circulação extracorpórea com uso de "shunt" intracavitário: 12 anos de experiência. Rev Bras Cir Cardivasc 1997; 12: $226-32$.

33 Fanning W J, Kakos G S, Willians T E Jr. - Reoperative coronary artery bypass grafting without cardiopulmonary bypass. Ann Thorac Surg 1993; 55: 486-9.

34 Loop F D, Lytle B W, Cosgrove D M et al. - Influence of the internal mammary-artery-graft of 10 -year survival and other cardiac events. N Engl J Med 1986; 314: $1-6$

35 Cameron A, Davis K B, Green G, Schaff H V - Coronary bypass surgery with internal-thoracic-artery grafts: effects on survival over a 15 -year period. $N$ Engl $J$ Med 1996; 334: 216-9.

36 Calafiore A M, Di Giammarco G, Teodori G et al. - Left anterior descending coronary artery grafting via left anterior small thoracotomy without cardiopulmonary bypass. Ann Thorac Surg 1996; 61: 1658-65.

37 Lobo Filho J G, Oliveira F M, Ciarline C et al. - Cirurgia de revascularização do miocárdio através de minitoracotomia anterolateral esquerda. Rev Bras Cir Cardiovasc 1996; 11: 143-7. 